Recepción: 27/05/2015

Aceptación: 29/07/2015

Leopoldo M. A. Godio

\title{
La Convención de las Naciones Unidas sobre el Derecho del Mar de 1982 y las actividades militares
}

\author{
The United Nations Convention on \\ the Law of the Sea and military activities
}

Resumen: Los océanos del mundo han jugado un papel crucial en el derecho internacional contemporáneo, facilitando la comunicación y proporcionando un importante progreso jurídico a partir de la III Conferencia de las Naciones Unidas sobre el Derecho del Mar, realizada entre 1973 y 1982, no sólo respecto de recursos vivos y no vivos, sino también, según expresó Boczek, como campo de batalla para innumerables conflictos armados y a modo de área para proyectar el poder naval con metas políticas utilizadas tanto para fines pacíficos como no pacíficos.

Palabras clave: derecho del mar; actividades militares; usos pacíficos; poder naval; relaciones internacionales

Abstract: The world oceans have played a crucial role in the contemporary international law, facilitating the communication and providing an important progress from the III United Nations Conference on the Law of the Sea held between 1973 and 1982, not only in respect of living and non-living resources, but have also served, stated Boczek, as a battleground for innumerable armed conflict and an area for projecting naval power with political goals used for peaceful as well non-peaceful purposes. In this paper, we will make a brief description of the development of military activities, his connections with the pacific purposes of the sea and we will later refer to some ideas that may be a starting point for an academic debate.

Keywords: law of the sea; military activities; pacific uses; naval power; international relations

\footnotetext{
$\stackrel{4}{4}$ Abogado y Magister en Relaciones Internacionales (Universidad de Buenos Aires, U.B.A.). Profesor de Derecho Internacional Público. Investigador adscripto del Instituto de Investigaciones Jurídicas y Sociales “Ambrosio L. Gioja” (Facultad de Derecho, UBA). Miembro del Instituto de Derecho Internacional de la Academia Nacional de Derecho y Ciencias Sociales de Buenos Aires. Miembro Titular de la Asociación Argentina de Derecho Internacional (A.A.D.I.) y del Instituto de Derecho Internacional del C.A.R.I., entre otras instituciones.

$\triangle$ leopoldogodio@derecho.uba.ar
} 



\section{Las actividades militares y la Convención de las Naciones Unidas sobre el Derecho del Mar de 1982}

\section{Introducción}

Durante siglos, el individuo utilizó las aproximadamente tres cuartas partes de la superficie del planeta con dos finalidades primordiales: la pesca y la navegación. Los mares fueron considerados durante mucho tiempo "bienes comunes" que trascendían la jurisdicción de cualquier Estado, tal como las pasturas medievales abiertas a los aldeanos que, luego de un tiempo y como respuesta a cambios productivos, tecnológicos y económicos fueron "cercados" provocando, con el transcurso de los siglos, una serie de controversias en torno a los tipos, extensiones, y determinación de distintos espacios marítimos sujetos a la jurisdicción Estatal.

La época señalada es perfectamente comprensible en un contexto dominado por el derecho internacional clásico en el que, desde mediados del siglo XIX hasta las primeras décadas del siglo XX, reinaba la libertad de los mares y las zonas de alta mar eran consideradas como res nullius y los reclamos de jurisdicción marítima eran prácticamente inexistentes. ${ }^{1}$

Incluso, desde una mirada de las relaciones internacionales, algunos autores sostienen que este régimen fue estrechamente asociado a los intereses y el poderío del principal país naval, el Reino Unido, al permitírsele dominar las periferias del mundo para garantizar, mediante la fuerza militar, la libertad del comercio y de los mares, dando lugar a la denominada Pax Britannica comprendida entre 1815 y 1914 (Keohane, R. \& Nye, J., 1988).

Lo anterior adquiere relevancia ya que, según Keohane y Nye, dos de las áreas claves de la Pax Britannica (y que persisten actualmente), han sido los asuntos monetarios y los espacios y recursos oceánicos. Sin embargo, es justo reconocer que se han producido cambios dramáticos en los patrones de comunicación y transporte, en la navegación marítima, en la pesca, en los mercados internacionales y en los sistemas financieros con una importancia intrínseca para los problemas maríti- 
mos que reúne, sin dudas, las condiciones óptimas de una interdependencia compleja a pesar de presentar aspectos abordables desde la teoría realista, gracias al papel de las armadas y prefecturas navales ya que, después de todo, aún patrullan los mares y, ocasionalmente, intentan reforzar los reclamos de jurisdicciones nacionales (Keohane, R. \& Nye, J., 1988, 89-90).

Lo anterior generó, parafraseando a Keohane, una reducción de la política de espacios y una disminución del aislamiento de los usuarios de los océanos, cuestión claramente evidenciada en la agenda política durante las negociaciones en la III Conferencia de las Naciones Unidas sobre el Derecho del Mar (en adelante "III Conferencia, indistintamente), desarrollada entre 1973 y 1982.

\section{Las "actividades militares" y el derecho del mar: una aproximación a su conceptualización}

La Convención de las Naciones Unidas sobre el Derecho del Mar (en adelante, "la Convención" o "la Convención de 1982", indistintamente), considerada una "Constitución para los Océanos" (Koh, T. B. 1983), representa la mayor obra destinada a una reforma integral del régimen, gozando de un éxito sin precedentes en la materia. En su conjunto, representa un equilibrio entre diferentes intereses competitivos en relación con los usos del mar (Keohane, R. \& Nye, J., 1988, 39-40), estableciendo un régimen de modo equitativo para La Zona y los recursos de los fondos marinos más allá de las jurisdicciones nacionales, resolviendo también una amplia serie de cuestiones afines, incluyendo la anchura del mar territorial y el paso a través de los estrechos utilizados para la navegación internacional, así como el problema de los derechos de soberanía sobre los recursos naturales y la prevención de la contaminación del medio marino.

Las actividades militares realizadas por los Estados reviste un tema complejo de vital importancia en la actualidad, especialmente, a partir de las controversias suscitadas entre los EEUU y China en la Zona Económica Exclusiva (en adelante, ZEE) de este último (Yao-Dong, Y. \& Wen-Jin, P. (2011).

El problema central de lo anterior radica en que la Convención no contiene normas sustanciales sobre la materia y ni siquiera se refiere, expresamente, a la aplicación del derecho internacional humanitario en este ámbito. Lo anterior permite pensar que los usos militares han sido uno de los aspectos "deliberadamente omitidos" durante el desarrollo de la III Conferencia de las Naciones Unidas sobre 
el Derecho del Mar (United Nations, 2009).

La negligencia a la que aludimos puede justificarse a partir de la inclusión, por parte de Arvid Pardo, el Representante Permanente de Malta ante la Organización de las Naciones Unidas (en adelante también, "ONU", indistintamente) que tuvo un rol determinante en los antecedentes que condujeron a la III Conferencia ${ }^{2}$, al escoger voluntariamente los problemas de los usos militares de los océanos y de las regiones polares de una lista de siete temas que fueron descuidados durante la Conferencia señalada, por tratarse de asuntos excesivamente políticos o delicados (Rauch, E., 1985).

Las potencias navales dominantes en aquel entonces, los EEUU y la URSS, se negaban sistemáticamente a negociar estos asuntos, tal se evidenció en la declaración del delegado norteamericano durante el debate Plenario de la Conferencia en 1976, al referirse al alcance del término "fines pacíficos" y expresar: "Cualquier limitación concreta de las actividades militares requerirá la negociación de un acuerdo detallado sobre el control de armamentos. La Conferencia no tiene ese fin y no está preparada para ese tipo de negociaciones. Cualquier intento de desviar la actuación de la Conferencia hacia una tarea tan compleja pondrá fin rápidamente a los actuales esfuerzos para negociar una convención sobre el derecho del mar..." (United Nations, 2009). En otras palabras, la declaración ilustra la gran sensibilidad que requería la construcción de la futura Convención mediante el consenso como método de negociación por las delegaciones allí presentes. ${ }^{3}$

De hecho, el término "usos militares" sólo aparece expresamente una vez en el texto de la Convención, incluyendo sus anexos, en el artículo 298 (1) (b), al enumerar las excepciones facultativas a los procedimientos obligatorios conducentes a decisiones obligatorias del sistema de solución de controversias relativas a la interpretación o aplicación de la Convención, dispuestas en la Parte XV, en los siguientes términos: “(...) Las controversias relativas a actividades militares, incluidas las actividades militares de buques y aeronaves de Estado dedicados a servicios no comerciales...". ${ }^{4}$

A pesar de lo anterior, algunas disposiciones de la Convención se refieren indirectamente a ciertos usos del mar que presentan, en su esencia, una naturaleza militar. Por ejemplo, al ocuparse del significado del paso inocente a través del mar territorial el artículo 19 menciona-entre las actividades perjudiciales para la paz, el buen orden o la seguridad del Estado ribereño-, cualquier ejercicio o prác- 
tica con armas de cualquier clase; cualquier acto destinado a obtener información en perjuicio de la defensa o la seguridad del Estado ribereño...; el lanzamiento, recepción o embarque de aeronaves; el lanzamiento, recepción o embarque de dispositivos militares (Naciones Unidas, 1982).

Según Vukas, esta Convención no aclara el significado del término "actividades militares" y que aquellas se limitarían, interpreta, a un solo propósito: aumentar la preparación de un Estado para la guerra, mediante buques de guerra o aeronaves militares o por buques y aeronaves del Estado destinados a fines no comerciales (Vukas, B., 1990).

En consecuencia, podríamos preguntarnos: ¿Cuáles son los posibles usos militares del mar en tiempos de paz? Siguiendo una enumeración formulada por Wolfrum, podemos agruparlas en algunas categorías:

1. Navegación sobre la superficie o en la columna de agua, incluyendo allí todas las actividades conexas a la navegación, sus maniobras preparatorias (tanto para la navegación como para la realización de ejercicios navales), la cooperación entre fuerzas navales, aéreas y terrestres de dos o más Estados, el control de los mares, la presencia naval y las tareas de disuasión, entre otras;

2. Emplazamiento de misiles en el fondo del mar con propósitos estratégicos;

3. Emplazamiento de dispositivos de vigilancia en el fondo del mar como, por ejemplo, los sistemas acústicos de detección;

4. Emplazamiento de un sistemas de armas en el fondo del mar con fines estratégicos o tácticos como, por ejemplo, minas magnéticas destinadas a buques de superficie o submarinos; $y$,

5. Investigación militar, incluyendo la prueba de armas en la superficie, la columna de agua o el lecho y su subsuelo marino. (Wolfrum, R., 1981).

La enumeración señalada resulta, a nuestro criterio, enunciativa no sólo por las propias características dinámicas del derecho internacional, sino también por las posibilidades que brinda el progreso tecnológico destinado a la guerra naval, especialmente el desarrollado por las potencias con intereses marítimos, como el caso de los EEUU, la Federación de Rusia y China, para men- 
cionar algunos (Bosco, J.,1997-1998).

No obstante, la Convención sí distingue entre las actividades militares y aquellas destinadas a garantizar el cumplimiento de ésta, en particular respecto de las normas de ejecución. En efecto, el artículo 298 ya referido, faculta en su párrafo $1^{\circ}$ las declaraciones de los Estados, formuladas por escrito, tendientes a no aceptar uno o varios de los procedimientos previstos en la sección anterior, es decir, las previstas en el artículo 287, respecto de las “(...) las controversias relativas a actividades encaminadas a hacer cumplir las normas legales respecto del ejercicio de los derechos soberanos o de la jurisdicción excluidas de la competencia de una corte o un tribunal con arreglo a los párrafos 2 o 3 del artículo 297". ${ }^{5}$

En definitiva, los párrafos señalados establecen las limitaciones a los procedimientos obligatorios de solución de controversias en los casos referidos a la investigación científica marina o la pesca. De tal modo, en caso que el Estado ribereño ejerza su competencia para hacer cumplir la Convención y sus disposiciones dictadas de conformidad con ella, ello no podría ser considerado como una actividad militar, aun cuando se realice por medio de buques de guerra o aeronaves militares.

\section{Otros antecedentes y argumentos utilizados en la construcción de una idea de actividades militares del mar}

A pesar de lo señalado con anterioridad, el origen convencional de los usos militares del mar no pueden atribuirse, al menos solamente, a la Convención de 1982. En efecto, existen importantes antecedentes vinculados a los fines pacíficos que, "transpolados" y con algunas adaptaciones necesarias al momento de su interpretación, permiten aproximarnos aún más a una correcta calificación de actividades militares, tal como fueron consideradas por las delegaciones presentes en la III Conferencia. A continuación recordaremos, cronológicamente, algunos de los precedentes que consideramos más relevantes, para luego dedicarnos a la III Conferencia y formular una breve referencia a los desafíos actuales.

\section{a) Las cláusulas sobre "fines pacíficos" en instrumentos internacionales anteriores a la Convención:}

El concepto de "fines pacíficos" fue empleado en distintos acuerdos internacionales como el Tratado Antártico de 1959 (en vigor desde 1961), que en su artículo 1 (1) declara a la Antártida como un territorio utilizado exclusivamente 
para fines pacíficos, entendiéndoselo a la manera de una prohibición de toda medida de naturaleza militar como, por ejemplo, el establecimiento de bases, la realización de maniobras militares e incluso, el ensayo con toda clase de armas ${ }^{6}$. Según Boczek, su redacción permite concluir que el término "exclusivamente con fines pacíficos" significa una total desmilitarización de la Antártida (Boczek, B., $1997)^{7}$. En otras palabras, la expresión "fines pacíficos" en este tratado excluye toda actividad militar y no sólo aquellas que sean manifiestamente violatorias del artículo 2 (4) de la Carta de las Naciones Unidas.

Otro acuerdo internacional que contiene la noción "fines pacíficos" es el Tratado sobre los principios que deben regir las actividades de los Estados en la exploración y utilización del espacio ultraterrestre, incluso la Luna y otros cuerpos celestes de 1969. El acuerdo señalado prohíbe, en el artículo IV, la disposición en órbita de armas nucleares u otros tipos de armas de destrucción masiva emplazadas en este espacio, incluyendo el establecimiento de bases, instalaciones y fortificaciones militares o efectuar ensayos con fines militares. ${ }^{8}$

Sin embargo, en el ámbito del derecho mar, la noción "fines pacíficos" aparece contenida en la "Declaración de Principios que regulan los fondos marinos y oceánicos fuera de los límites de la jurisdicción nacional”, proclamada en 1970 por la Asamblea General de la ONU, AG 2749 (XXV) y que consagra a aquellas zonas y sus recursos como "patrimonio común de la humanidad" quedando, asimismo, reservadas para fines pacíficos y sin perjuicio de las medidas que se puedan adoptar dentro de las negociaciones de desarme.

Justamente, en 1971 y también bajo los auspicios de la ONU, se suscribió el "Tratado sobre Prohibición de Emplazar Armas Nucleares y otras Armas de Destrucción en Masa en los Fondos Marinos y Oceánicos y su Subsuelo" que carece, en su parte operativa, de referencias expresas a fines pacíficos y sólo se refiere a aquellos en su preámbulo al afirmar que estas áreas quedarán reservadas, toda vez que “(...) la prevención de la carrera de armamentos nucleares en los fondos marinos y oceánicos favorece la causa del mantenimiento de la paz mundial, reduce las tensiones internacionales y refuerza las relaciones amistosas entre los Estados... constituye un paso hacia la exclusión de los fondos marinos y oceánicos y su subsuelo de la carrera de armamentos...".

Sobre este preámbulo, Boczek observa que una interpretación unificada nos convencería de que el último tratado señalado sólo constituye un paso hacia la 
exclusión del lecho y el subsuelo marino en la carrera armamentista, obligándonos a concluir, respecto de los "fines pacíficos", que este aspecto sólo se proyecta como una meta hacia el futuro (Boczek, B., 1989).

De este modo no aparecen en los antecedentes a la III Conferencia claras demostraciones sobre la existencia de una interpretación respecto de la noción "fines pacíficos" ya como lo refleja el contexto y circunstancias de cada instrumento en cuestión (Boczek, B., 1997)9. Para completar lo anterior, Wolfrum afirma que esta expresión es utilizada en diversos tratados internacionales sin brindar una respuesta clara en cuando a su significado para la Convención de 1982 (Wolfrum, R., 1998).

\section{b) Las disposiciones sobre "fines pacíficos" en la Convención de 1982. Su relación con la Carta de la Organización de las Naciones Unidas:}

No obstante lo anterior, y como cara opuesta de la misma moneda medalla referida a las "actividades militares", la propia Convención contiene varias disposiciones relativas al uso de los mares y océanos con fines pacíficos que permitirían aproximarnos a una idea más acabada de lo que podríamos llegar a considerar como "actividades militares permitidas". Ello puede inferirse a partir de Vukas, al afirmar que las "(...) pocas y ambiguas disposiciones sobre los usos militares del mar se deben a la actitud de la gran mayoría de los participantes de la Tercera Conferencia que... trataron de minimizar las diferencias en esa cuestión. Sin embargo... muchas de las disposiciones de la Convención... se aplican a un número de actividades, independientemente de su carácter civil o militar" (Vukas, B., 1990) (Caminos, H., 2001).

Si bien muchos de los asuntos tratados de la Convención de 1982 poseen antecedentes en las Convenciones de Ginebra de 1958 y 1960, ninguna de éstas contiene referencia alguna a los fines o usos pacíficos del mar. A pesar de ello, y tal como hemos adelantado, la idea sí estuvo presente, al menos inicialmente, desde el comienzo del proceso de reforma que condujo a la convocatoria de la III Conferencia, tendiente a obtener una regulación completa e integral de los usos de mares y océanos.

Ya en 1967, con fundamento en la iniciativa de Arvid Pardo, se incorporó el examen de la "Cuestión de la reserva exclusiva para fines pacíficos de los fondos marinos y oceánicos y de su subsuelo en alta mar fuera de los límites de la jurisdicción nacional actual y del empleo de sus recursos en beneficio de la Humani- 
dad" al temario base de debates. Como resultado del trabajo de dos comités establecidos por la Asamblea General para estudiar esta cuestión, en 1967 y 1968, ésta adoptó en 1970 la Resolución 2749 (XXV) que, como expresáramos con anterioridad, declaró a los fondos marinos y oceánicos, junto a su subsuelo, reservados para fines pacíficos.

Uno de los tópicos de la lista sometida a la III Conferencia fue el ítem 22, denominado "Utilización con fines pacíficos del espacio oceánico, zonas de paz y seguridad", que se asignó al Plenario de la Conferencia. Allí, en el debate que tuvo lugar en 1976 prevaleció una opinión a considerar que los problemas referidos a los usos pacíficos del mar y su desmilitarización debían ser discutidos en otro foro (Vukas, B., 1990). No obstante, varias delegaciones propusieron su inclusión mediante un párrafo sobre usos pacíficos en el Preámbulo que, finalmente, fue redactado en 1980 e incorporado al texto definitivo de la Convención de $1982 .{ }^{10}$

Luego de la adopción del texto, encontramos que el mismo posee varios artículos que regulan los usos pacíficos aplicables a zonas y actividades marítimas específicas, a saber:

a) el artículo 88, al disponer que la alta mar será utilizada exclusivamente con fines pacíficos;

b) el artículo 58 (2) que aplica, mutatis mutandi, al artículo anterior a la ZEE;

c) el artículo 141, al reconocer que la Zona Internacional de los Fondos Marinos estará abierta a la utilización exclusivamente con fines pacíficos por todos los Estados;

d) el artículo 143 al señalar que la investigación científica marina en la Zona referida anteriormente se realizará también, exclusivamente, con fines pacíficos;

e) el artículo 147 (2) (d), que regula las condiciones y los fines pacíficos a las que estarán sujetas las instalaciones utilizadas para realizar actividades en la Zona;

f) el artículo 155 (2) por cuanto reconoce a la Conferencia de Revisión de la Parte XI, el velar por el mantenimiento del principio de utilización pacífico de la Zona; ${ }^{11}$ 
g) el artículo 240 (a), destinado a enumerar los principios generales para la realización de la investigación científica marina; $y$,

h) el artículo 246 (3), al establecer que los Estados ribereños, en circunstancias normales, otorgarán su consentimiento para que otros Estados u organizaciones internacionales realicen proyectos de investigación científica en su ZEE o en su plataforma continental "exclusivamente con fines pacíficos".

Además de las normas precedentes, aplicables a zonas y actividades marítimas específicas, el artículo 301 de la Convención, referido a la utilización del mar con fines pacíficos, e incluido en la Parte XVI bajo el título "Disposiciones Generales", expresa que "Al ejercer sus derechos y cumplir sus obligaciones de conformidad con esta Convención, los Estados Partes se abstendrán de recurrir a la amenaza o al uso de la fuerza contra la integridad territorial o la independencia política de cualquier Estado o en cualquier otra forma incompatible con los principios de derecho internacional incorporados en la Carta de las Naciones Unidas".

Los debates previos a su redacción, por parte de los delegados negociadores en la III Conferencia, se presentaron en el plenario del año 1976 y en tan sólo tres sesiones bajo el tema "Utilización con fines pacíficos del espacio oceánico: zonas de paz y seguridad" (Naciones Unidas, 1973-1982) lograron incorporar varias cláusulas referidas a "fines pacíficos", tendientes a su inclusión en las disposiciones que luego fueron reconocidos en los artículos que ya señalados (Naciones Unidas, 1973-1982).

En el debate, se plantearon básicamente dos posiciones divergentes acerca del significado de "usos pacíficos". La primera fue formulada por los EEUU, en representación de las potencias marítimas, cuyo delegado expresó que el mismo “(...) no excluye, por supuesto, las actividades militares en general... la realización de actividades militares con fines pacíficos está totalmente de acuerdo con la Carta de las Naciones Unidas y con los principios del derecho internacional"; la segunda, sintetizada por Ecuador, en representación de los Estados que no eran potencia en la materia, expresó como réplica que “(...) ya se ha reconocido en muchos órganos y acuerdos internacionales que la utilización del espacio oceánico para fines exclusivamente pacíficos debe significar la desmilitarización completa y la exclusión del espacio oceánico de todas las actividades militares" (Naciones Unidas, 1973-1982).

En definitiva, la solución al debate se tradujo en la inserción de un párrafo en 
el Preámbulo de la Convención, redactado por el Presidente de la III Conferencia y luego de las sesiones plenarias oficiosas mediante el recurso del consenso, referido a los usos con fines pacíficos de los mares y océanos. ${ }^{12}$

Por otra parte, el artículo 301 de la Convención posee su origen en una propuesta oficiosa presentada por Costa Rica, Ecuador, El Salvador, Filipinas, Pakistán, Perú, Portugal, Senegal, Somalia y Uruguay. Al respecto, cabe recordar que, como disposición general, el ámbito de aplicación del artículo señalado comprende todos los espacios y actividades marítimas aunque el mismo texto contiene una remisión a la Carta de las Naciones Unidas y los principios de derecho internacional incorporados a ésta en los términos del artículo 2 (4) y concordantes de la Carta (Klein, N., 2004).

De lo anterior podemos afirmar que, en principio, el artículo 301 de la Convención de 1982 podría ser entendido como una prohibición de toda actividad militar, o bien como una prohibición de los actos comprendidos en el artículo 2 (4) de la Carta fundante de las Naciones Unidas.

Por su parte, Wolfrum se encarga de analizar los alcances de la norma y considera que, debido a la vaguedad del artículo 2 (4) de la Carta, resulta difícil interpretar su "exacto" contenido. El autor se formula preguntas como: “¿Cuál es el significado preciso de la expresión 'incompatible con los principios de derecho internacional incorporados a la Carta de las Naciones Unidas’?”; “Qué significa el término 'amenaza o uso de la fuerza'?; y ¿Cómo se relaciona con el término 'amenaza para la paz, quebrantamiento de la paz o acto de agresión' en el artículo 39 y 51 de la Carta de la ONU?. El jurista señalado agrega que ninguna de estas cuestiones ha sido aclarada en la Definición de Agresión, adoptada por la Asamblea General en 1974 (AG 3314 (XXIX)), ya que esta última sólo contempla la prohibición de los ataques en los mares territoriales, mientras que la ZEE y la plataforma continental, sujetas a derechos de soberanía, quedarían sin protección (Wolfrum, R.,1981).

No obstante, la Resolución 3314 enumera algunos actos que serían calificados como agresión, por ejemplo, el bloqueo de puertos o costas de un Estado por las fuerzas armadas de otro (art. 3.c) y el ataque por las fuerzas armadas de un Estado contra las fuerzas terrestres, marítimas o aéreas de otro (art. 3.d), aunque interpretamos que, sobre este punto deberá tenerse en consideración el Manual de San Remo sobre el Derecho Internacional Aplicable a los Conflictos Armados en el Mar de 1994, junto con la práctica de los Estados en la materia como, por ejem- 
plo, la realizada por las fuerzas navales de los EEUU detalladas en el Commander's Handbook on the Law of Naval Operations del año $2007 .{ }^{13}$

En definitiva, interpretamos que la propuesta original del 2 (4) de la Carta se refiere sólo a los propósitos de las Naciones Unidas, mientras que el texto del artículo 301 de la Convención de 1982 no sólo incluye los principios reconocidos en el Capítulo I de la Carta, sino también los principios de derecho internacional que se encuentren en otras disposiciones como, en particular, el derecho inmanente de legítima defensa individual o colectiva, según el caso, reconocido a favor de los Miembros de la ONU en el artículo 51. Según Rauch, la consecuencia es que "todas las actividades navales en los océanos [realizadas] en preparación para la defensa individual o colectiva son compatibles con la Convención y deben considerarse como 'uso pacífico'... " (Rauch, E.,1985).

Lo anterior nos permite coincidir con Vukas, al afirmar que el artículo 301 de la Convención de 1982 representa la aplicación explícita, al derecho del mar, de algunos principios básicos del derecho internacional general y de la Carta de las Naciones Unidas, particularmente del artículo 2 (4), en el sentido que el mismo no limita ni prohíbe ninguna actividad militar particular en tanto aquella no sea contraria a los principios mencionados en el artículo de la Convención de 1982 señalado (Vukas, B., 1990).

\section{c) La aparición de espacios o situaciones no contempladas en la III Conferencia:}

A partir de las ideas anteriormente desarrolladas, comenzamos a vislumbrar cómo la práctica de los Estados genera, de conformidad con las propias características del derecho internacional público, la aparición de un controvertido y actual debate académico sobre la existencia de espacios marítimos no regulados por la Convención de 1982. Al respecto, autores como Treves manifiestan su preocupación por algunos de ellos que, en la materia que nos interesa, pueden tener como efecto situaciones que involucran directa o indirectamente el desarrollo de actividades militares como las áreas marinas protegidas o los espacios de protección ambiental que, posteriormente, puedan transformarse o justificarse como en una zona de seguridad militar (Treves, T. , 2012), tal como ocurrió, en cierta medida, con la controversia del buque de Greenpeace, el Arctic Sunrise, suscitada entre los Países Bajos y la Federación de Rusia, en octubre de 2013. ${ }^{14}$ 


\section{Conclusiones}

En el presente trabajo, hemos intentado aclarar algunos aspectos que presenta la expresión "actividades militares" en la Convención de las Naciones Unidas sobre el Derecho del Mar de 1982, advirtiendo que estas pueden involucrar posibles acciones habilitadas por el derecho internacional como la navegación de buques de guerra y el sobrevuelo de aeronaves con idéntica naturaleza, la investigación científica marina realizada por personal militar, ejercicios y maniobras militares que pueden incluir pruebas de armamentos, entre otros.

Sin embargo, otras situaciones de "actividades militares" requieren de una mirada más detallada, dependiendo de cada caso puntual, al menos en cuanto al derecho del mar se refiere, a partir de la consideración de los "usos pacíficos" que hemos planteado y su vinculación con el artículo 2 (4) de la Carta de las Naciones Unidas.

Finalmente, no deseamos concluir sin destacar que la indudable aparición de controversias recientes en todo el mundo, cada una con características particulares como, por ejemplo, las señaladas en el Mar de China, merecen un estudio. Por otra parte, también es cierto que los negociadores de la III Conferencia lograron a nuestro entender, en un sutil equilibrio de codificación y desarrollo progresivo, el reconocimiento de los intereses de la comunidad internacional, tanto de los Estados ribereños y como de los Estados que adviertan o realicen actividades que puedan ser calificadas como "militares" más allá de los espacios adyacentes a su mar territorial.

No obstante, consideramos que el tema no se agotaría necesariamente en la calificación de una actividad como "militar", y su consiguiente habilitación o prohibición, sino que presenta posteriores desafíos a partir de las excepciones facultativas de la Parte XV de la Convención de 1982, referida a la solución pacífica de controversias, a modo de instar a las partes al respeto mutuo en el marco del derecho internacional, considerando los artículos 2 (3) y 33 de la Carta de las Naciones Unidas. 


\section{Referencias}

Armas Pfirter, F. (1994). El Derecho Internacional de Pesquerías y el frente maritimo del Río de la Plata. Buenos Aires: Consejo Argentino para las Relaciones Internacionales.

Blank, L. (2012). Complex legal frameworks and complex operational challenges: navigating the applicable law across the continuum of military operations. Emory International Law Review, (26), 87-135.

Bosco, J. (1997-1998). The International Law Implications of China's Military and Missile Exercises in the Taiwan Strait under the 1982 United Nations Law of the Sea Convention and the United Nations Charter. Chinese (Taiwan) Yearbook of International Law and Affairs, (16), 50-58.

Boczek, B. (1989). Peaceful purposes provisions of the United Nations Convention on the Law of the Sea. Ocean Development \& International Law, (20), 363.

Boczek, B. (1997). The peaceful reservation of the United Nations Convention on the Law of the Sea. En Broghese, E. (ed.) Peace in the Oceans. The Proceedings of Pacem in Maribus XXII, Unesco.

Caminos, H. (comp.) (2001). Law of the Sea, Dartmouth, Ashgate.

Daly, J. (2014). Russia Convinces 'Caspian Five' to Bar Foreign Militaries From the Caspian”. Eurasia Daily Monitor, (11)83, 3-5.

Geng, J. (2012). The Legality of Foreign Military Activities in the Exclusive Economic Zone under UNCLOS". Merkourios, (28)74, 22-30.

Hayashi, M. (2012). Military Activities in the Exclusive Economic Zones of Foreign Coastal States. International Journal of Marine \& Coastal Law, (27)4, 795-803.

Hong, N. (2010). Law and Politics in the South China Sea. Assessing the Role of UNCLOS in Ocean Dispute Settlement. Edmonton: University of Alberta. 
Janis, M. (1977). Dispute Settlement in the Law of the Sea Convention: The Military Activities Exception. Ocean Development \& International Law, (4)1, 51-65.

Keohane, R. \& Nye, J. (1988). Poder e interdependencia. La politica mundial en transición. Buenos Aires: GEL.

Klein, N. (2004). Dispute Settlement in the UN Convention on the Law of the Sea. Cambridge: Cambridge University Press.

Kline, R., (2013). The pen and the sword: the people's Republic of China's effort to redefine the Exclusive Economic Zone through maritime lawfare and military enforcement. Military Law Review, (216), 122-169.

$\mathrm{KoH}, \mathrm{T}$. (1983). A Constitution for the Oceans. En United Nations, The Law of the Sea - Official Text of the United Nations Convention on the Law of the Sea with Annexes and Index. New York: UN

Letts, D. (2005). Recent Australian Experience of the Law of the Sea and Military Operations", Australian Year Book of International Law, (24), 129-144.

Neri, K. (2012). The Use of Force by Military Vessel Protection Detachments. Military Law and Law of War Review, (51)1, 73-96.

Pedrozo, R. (2010). Preserving Navigational Rights and Freedoms: The Right to Conduct Military Activities in China's Exclusive Economic Zone. Chinese Journal of International Law, (9)1, 9-29.

Pitsoulis, A. \& Schwuchow, S. (2014). Coercion, Credibility, and Mid-Air Interceptions of Military Planes. Peace Economics, Peace Science, \& Public Policy, (20)4, 697-707.

Pirtle, C. (2000). Military Uses of Ocean Space and the Law of the Sea in the New Millennium. Ocean Development \& International Law, (31)1/2, 7-45.

Ranganathan, S. (2014). Strategically Created Treaty Conflicts and the Politics of International Law. Cambridge: Cambridge University Press. 
Rauch, E. (1985). Military Uses of the Oceans. German Yearbook of International Law, 28, 230-231.

Rosen, M. (1995). Military Mobility and the 1982 UN Law of the Sea Convention. Georgetown International Environmental Law Review, (7)3, 717-724.

Sienho, Y. (2010). Sketching the Debate on Military Activities in the EEZ: An Editorial Comment. Chinese Journal of International Law, (9)1, 1-7.

Shyam, M. (1985). The U.N. Convention on the Law of the Sea and military interests in the Indian Ocean. Ocean Development \& International Law, (15), 147-170.

Stephens, D. (1999). The Impact of the 1982 Law of the Sea Convention on the Conduct of Peacetime Naval/Military Operations. California Western International Law Journal, (29), 283-311.

Treves, T. (2012). The High Seas as Potential Exclusive Economic Zones in the Mediterranean. En Kohen, M., Kolb, R. \& Liva Tehindrazanarivelo, D. (eds.), Perspectives of International Law in the 21st Century. Liber Amicorum Professor Christian Dominicé in Honour of his 80th Birthday, (175-189), Leiden/Boston, Martinus Nijhoff.

Truver, S. (1985). The law of the sea and the military use of the oceans in 2010. Louisiana Law Review, (45), 1221-1247.

Vadi, V. (2009). International Law and the Uncertain Fate of Military Sunken Vessels. Italian Yearbook of International Law, (19), 253-278.

Van Dyke, J. (2004). Military ships and planes operating in the exclusive economic zone of another count. Marine Policy, 28(1), 29-39.

Von Heinegg, W. (2000). Naval Blockade. En Schimitt, M. (ed.), International Law Across the Spectrum of Conflict: Essays in Honor of Professor L. C. Green on the Occasion of his Eightieth Birthday, (203-230). Newport: Naval War College.

Vukas, B. (2004). L' utilization pacifique de la mer, dénucléarisation et désarmement. En: Vukas, B., Law of the Sea: Selected Writings. (157-204), Leiden: Brill. 
Vukas, B. (1990). Military Uses of the Sea and the United Nations Law of the Sea Convention. En: Vukas B. (comp.), Essays on the New Law of the Sea 2, (401427). Zagreb: Sveucilisna Naklada Liber.

Wang, J. (2012). Freedom of and Restraints on Maritime Military Activities: A Study Based on UNCLOS. Journal of Southern Yangtze University: Humanities \& Social Sciences, (11)1, 43-50.

Wilson, B. (2010). An Avoidable Maritime Conflict: Disputes Regarding Military Activities in the Exclusive Economic Zone. Journal of Maritime Law \& Commerce, (41), 421-437.

Wolfrum, R. (1998). Military Activities on the High Seas: What Are the Impacts of the U.N. Convention on the Law of the Sea. En: Schmitt, M. and Green, L. (eds.), International Law Studies, 71, The Law of Armed Conflict: Into the Next Millenium, (501-513). Newport, RI: Naval War College.

Wolfrum, R. (1981). Restricting the Use of the Sea to Peaceful Purposes: Demilitarization in Being?. German Yearbook of International Law, 24, 205-206.

Yao-Dong, Y. \& Wen-Jin, P. (2011). Legal Study on Military Activities in the EEZ : With a Focus on Foreign Military Activities in the EEZ of P. R. China, KMI International Journal. of Maritime Affairs and Fisheries / KMI International Journal. of Maritime Affairs and Fisheries, 163-181.

Zedalis, R. (1984). Foreign state military use of another state's continental shelf and international law of the sea. Rutgers Law Journal, (16), 1-113.

Zhang, H. (2010). Is It Safeguarding the Freedom of Navigation or Maritime Hegemony of the United States? - Comments on Raul (Pete) Pedrozo's Article on Military Activities in the EEZ", Chinese Journal of International Law, (9)1, 31-47.

Zwanenburg, M. (2012). Military Vessel Protection Detachments: The Experience of the Netherlands. Military Law and Law of War Review, (51)1, 97-116. 


\section{Documentos y convenciones citadas (ordenados cronológicamente)}

Tratado Antártico. Disponible en http://www.ats.aq/s/ats.htm

Tratado sobre los principios que deben regir las actividades de los Estados en la exploración y utilización del espacio ultraterrestre, incluso la Luna y otros cuerpos celestes.

United Nations, Third United Nations Conference on the Law of the Sea, 1973-1982. Documents of the Conference, First (New York, 3-15 December 1973) and Second (Caracas, 20 June to 29 August 1974) Sessions A/CONF.62.

Note Verbale of 17 August 1967 from Malta to UN Secretary-General, UN GAOR, 22nd Session, UN Doc. A/6695 (1967).

Convención de Naciones Unidas sobre el Derecho del Mar, adoptada el 30 de abril de 1982, United Nations Treaty Series, Vol. 1833.

Manual de San Remo sobre el Derecho Internacional Aplicable a los Conflictos Armados en el Mar, 1994.

The Commander's Handbook on the Law of Naval Operations, Edition July 2007.

\section{Notas}

${ }^{1}$ Las controversias en espacios marítimos en material de pesca no eran numerosas. Sin embargo, podemos mencionar que en 1905 Uruguay había apresado un buque británico por pescar en aguas jurisdiccionales que, en aquel entonces, se encontraban controvertidas. Posteriormente, y ante la protesta diplomática del Estado de pabellón, se procedió a su liberación. Keohane, R. \& Nye, J. (1988), Poder e interdependencia. La política mundial en transición.

${ }^{2}$ Cabe recordar que fue el autor del discurso pronunciado, el 17 de agosto de 1967, ante la Asamblea General alentando al establecimiento de un régimen internacional para el lecho y el subsuelo marino más allá de las jurisdicciones nacionales. Conf. Note Verbale of 17 August 1967 from Malta to UN Secretary-General, UN GAOR, 22nd Session, UN Doc. A/6695 (1967). 
${ }^{3}$ Su idea se encuentra como apéndice al Reglamento de la III Conferencia: "Teniendo presente que los problemas del espacio oceánico están estrechamente relacionados entre sí y deben considerarse como un todo, y la conveniencia de adoptar una Convención sobre el Derecho del Mar que logre la máxima aceptación posible. La Conferencia debe hacer todos los esfuerzos posibles para que los acuerdos sobre los asuntos de fondo se tomen por consenso, y dichos asuntos no deberán someterse a votación hasta tanto no se hayan agotado todos los esfuerzos por llegar a un consenso". Conf. United Nations (2009), Third United Nations Conference on the Law of the Sea, 1973-1982. Documents of the Conference, Volume I, Doc.A/CONF. 62/SR 19. Al respecto, cabe mencionar la aclaración que realiza Armas Pfirter al sostener que “(...) aunque la Convención se negoció de acuerdo al consenso y 'package deal', para tratar de lograr un acuerdo general susceptible de aceptación universal, finalmente se adoptó por votación”. Conf. Armas Pfirter, F. (1994), El Derecho Internacional de Pesquerías y el frente marítimo del Río de la Plata, Buenos Aires, Consejo Argentino para las Relaciones Internacionales, 74.

${ }^{4}$ Conf. Convención de Naciones Unidas sobre el Derecho del Mar, adoptada el 30 de abril de 1982, United Nations Treaty Series, Vol. 1833, art. 298 (1) (b). Para un análisis profundizado de este tema ver Janis, M. (1977), "Dispute Settlement in the Law of the Sea Convention: The Military Activities Exception”, Ocean Development \& International Law, Vol. 4 Issue 1, 51-65. the United Nations Charter", Chinese (Taiwan) Yearbook of International Law and Affairs, Vol. 16, 50-58.

${ }^{5}$ Conf. Convención de Naciones Unidas sobre el Derecho del Mar, adoptada el 30 de abril de 1982, United Nations Treaty Series, Vol. 1833, art. 298 (1) (b). Cabe destacar que las declaraciones se encuentran permitidas con los alcances previstos en el artículo 310 de la Convención y normas conexas, siempre que no equivalgan a entenderse como reservas, prohibidas en el artículo 309 de la misma, como evidente consecuencia del consenso como método de negociación.

${ }^{6}$ El norma referida dispone, textualmente: "La Antártida se utilizará exclusivamente para fines pacíficos. Se prohíbe, entre otras, toda medida de carácter militar, tal como el establecimiento de bases y fortificaciones militares, la realización de maniobras militares, así como los ensayos de toda clase de armas".

${ }^{7}$ Conf. Boczek, B. (1997), "The peaceful reservation of the United Nations Convention on the Law of the Sea", En: Broghese, E., Peace in the Oceans. The Proceedings 
of Pacem in Maribus XXII, (93), París, Unesco. El autor agrega ejemplos de otras áreas desmilitarizadas como el tratado relativo a Spitzbergen, del 9 de Febrero de 1920, y la "Convención sobre la Neutralización y no Fortificación de las islas Aaland" de 1921, firmado por Dinamarca, Estonia, Finlandia, Francia, Alemania, Italia, Letonia, Polonia, Suecia y Reino Unido en Ginebra el 20 de Octubre de 1921.

${ }^{8}$ La norma expresa, de modo textual: "Los Estados Partes en el Tratado se comprometen a no colocar en órbita alrededor de la Tierra ningún objeto portador de armas nucleares ni de ningún otro tipo de armas de destrucción en masa, a no emplazar tales armas en los cuerpos celestes y a no colocar tales armas en el espacio ultraterrestre en ninguna otra forma.

La Luna y los demás cuerpos celestes se utilizarán exclusivamente con fines pacíficos por todos los Estados Partes en el Tratado. Queda prohibido establecer en los cuerpos celestes bases, instalaciones y fortificaciones militares, efectuar ensayos con cualquier tipo de armas y realizar maniobras militares. No se prohíbe la utilización de personal militar para investigaciones científicas ni para cualquier otro objetivo pacífico. Tampoco se prohíbe la utilización de cualquier equipo o medios necesarios para la exploración de la Luna y de otros cuerpos celestes con fines pacíficos".

${ }^{9}$ Conf. Boczek, B. (1997), "The peaceful reservation of the United Nations Convention on the Law of the Sea". En: Broghese, E., Peace in the Oceans. The Proceedings of Pacem in Maribus XXII, (53), París, Unesco. Otros instrumentos, como el "Tratado de No Proliferación de armas nucleares" de 1968, o el Estatuto del Organismo Internacional de la Energía Atómica, aprobado el 23 de octubre de 1956 por la Conferencia sobre el Estatuto del Organismo Internacional de Energía Atómica, celebrada en la Sede de las Naciones Unidas. Entró en vigor el 29 de julio de 1957 y utiliza el concepto de "usos pacíficos", aunque sin explicar su significado. En el orden regional, el Tratado para la Prohibición de Armas Nucleares en América Latina y el Caribe (más conocido como Tratado de Tlatelolco), de 1967, limita el uso de armas nucleares en América Latina y el Tratado de Raratonga, de 1985, establece una zona libre de armas nucleares en el pacífico sur.

${ }^{10} \mathrm{El}$ cuarto párrafo del Preámbulo, reconoce la conveniencia de establecer por medio de la Convención un orden jurídico que “(...) promueva los usos con fines pacíficos de los mares y océanos".

${ }^{11} \mathrm{Al}$ respecto, cabe destacar que el Acuerdo relativo a la aplicación de la Parte XI de 
la Convención de las Naciones Unidas sobre el Derecho del Mar de 10 de diciembre de 1982" (AGNU, Res. 48/623), aprobado el 28 de julio de 1994 (en vigor desde el 28 de julio de 1996, actualmente con 141 ratificaciones o adhesiones), declaró no aplicable la Conferencia de Revisión pero mantener los principios del artículo 155 (2), para el caso de reformas.

${ }^{12}$ Véase el informe del Presidente sobre la labor del Plenario de la Conferencia en la sesión oficiosa concerniente al Preámbulo. Véase Doc. A/CONF.62.L.49 y add. 1 y 2, en United Nations, Third United Nations Conference on the Law of the Sea, 19731982. Documents of the Conference, "Report of the President on the work of the informal plenary meeting of the Conference on the preamble", Volume XIII, pp. 78-82. En sentido estricto, fue el representante de Perú, Arias Schreiber, quien incentivara el debate y, posteriormente Yankov, de Bulgaria, quien propuso que en el Preámbulo de la Convención figurase un párrafo afirmando que la utilización con fines pacíficos del espacio oceánico "es el principio fundamental que rige las actividades de los Estados en su utilización de los mares y en la exploración y explotación de sus recursos". Ibidem, Volume V, p. 79 y ss.

${ }^{13}$ Sobre bloqueos navales ver von Heinegg, W. "Naval Blockade", in Schimitt, M. (ed.), International Law Across the Spectrum of Conflict: Essays in Honor of Professor L. C. Green on the Occasion of his Eightieth Birthday, (203-230), Newport, Naval War College.

${ }^{14}$ Conf. Arctic Sunrise Arbitration (Netherlands v. Russia), PCA. Disponible en http://www.pca-cpa.org/showpage.asp?pag_id=1556 (consulta el 15/04/2015). Ver también The Arctic Sunrise Case (Kingdom of the Netherlands v. Russian Federation), ITLOS, Provisional Measures, Order of 22 November 2013. 